\title{
Estimación de Sincrofasores en Redes Eléctricas Inteligentes: de Modelos a Restricciones de Diseño
}

\author{
Francisco Messina $^{\dagger 1}$, Leonardo Rey Vega ${ }^{\dagger * 2}$ y Cecilia G. Galarza ${ }^{\dagger * 3}$ \\ ${ }^{\dagger}$ Facultad de Ingeniería, Universidad de Buenos Aires \\ $1,2,3$ \{fmessina, lrey, cgalar\}@fi.uba.ar \\ *Centro de Simulación Computacional, CONICET
}

Recibido: 02/08/17; Aceptado: 12/09/17

\begin{abstract}
We first present a detailed review of two popular classes of model based synchrophasor estimators. They are the discrete Fourier transform (DFT) and the Taylor-Fourier transform (TFT) class estimators, which arise from constant or polynomial phasor models, respectively. Limitations of these techniques, arising from the models themselves, are exposed. These limitations make the original DFT and TFT approaches unfitted for situations where the phasor can not be properly described in such simple terms. This issue can be addressed with the recently introduced convex semi-infinite programming (CSIP) based estimators, which are also reviewed. In particular, we present the constraints associated with the tests which are most critical for the model based methods, showing how to precisely control the estimators performance in those cases. Finally, we show that the DFT and TFT estimators are particular instances of the CSIP estimator, so that there exists a connection between these two apparently different approaches. This opens the door for future analyses and developments.
\end{abstract}

Resumen- Presentamos en primer lugar una reseña de dos populares clases de métodos de estimación de sincrofasores. Ellas son la clase de métodos basados en la transformada de Fourier discreta (DFT) y la transformada de Taylor-Fourier (TFT), que surgen de un modelo de fasor constante y polinómico, respectivamente. Se exponen las limitaciones de estos métodos, que surgen por los propios modelos asumidos, lo cual los hace poco apropiados en situaciones donde el fasor no puede representarse en términos tan simples. Este problema puede resolverse con los estimadores basados en optimización convexa semi-infinita (CSIP), un enfoque novedoso que también es descripto. En particular, enfatizamos las restricciones asociadas con las situaciones más críticas para los métodos basados en modelos, mostrando cómo controlar precisamente el desempeño de los estimadores en dichos casos. Finalmente, mostramos que los estimadores DFT y TFT son instancias particulares del estimador CSIP, de modo existe una conexión entre estos dos enfoques aparentemente diferentes. Esto abre la puerta para futuros análisis y desarrollos.

\section{INTRODUCCIÓN}

\section{A. Contexto}

En los últimos años ha cobrado un gran interés, principalmente por su gran impacto potencial a nivel mundial, el paradigma de red eléctrica inteligente (en inglés Smart Grid o SG) [1]. Una SG busca alcanzar altos niveles de eficiencia y confiabilidad mediante la integración de distintos recursos como sensores inteligentes para monitoreo, fuentes de energía renovables, generación distribuida de energía, control robusto y automático, seguridad cibernética, etc. La confluencia de estos elementos involucra diversas áreas de ingeniería eléctrica y electrónica, así como áreas de ciencias de la computación.

Las unidades de medición fasorial (PMUs) son una componente fundamental de las SGs [2] pues permiten obtener prácticamente en tiempo real los parámetros más importantes de las señales de tensión y corriente en nodos críticos de la red eléctrica. Concretamente, dichos parámetros son: amplitud y fase (o conjuntamente fasor), frecuencia, y tasa de variación de frecuencia (ROCOF). La gran ventaja de esta tecnología, con respecto a los sistemas tradicionales, radica en que las mediciones de los PMUs están sincronizadas al estándar de tiempo UTC, típicamente por medio de un receptor GPS. Esta característica es lo que permite integrar en forma coherente mediciones de PMUs en grandes regiones geográficas, lo cual aporta información muy valiosa de la red eléctrica. De hecho, dicha información tiene un impacto directo en las diversas aplicaciones de estos sistemas de medición de gran área (WAMS) [3].

Idealmente, las señales de tensión son sinusoidales puras de frecuencia 50 o $60 \mathrm{~Hz}$ (dependiendo del país), pero en la práctica existirán pequeñas perturbaciones con respecto a los parámetros nominales que es importante observar periódicamente. Estas perturbaciones pueden ser, por ejemplo, corrimientos en la frecuencia, modulaciones en amplitud o en fase, rampas en frecuencia, saltos en amplitud o en fase, etc. A su vez, la señal puede estar contaminada por armónicas, interarmónicas y ruido. Otro requisito importante es que la latencia de las mediciones debe ser suficientemente pequeña, lo cual es particularmente crítico en aplicaciones de control y protección. Por esta razón, las mediciones de los PMUs deben satisfacer un conjunto de especificaciones estrictas, que están establecidas en el estándar de sincrofasores IEEE Std. C37.118.1-2011 [4] y su posterior enmienda IEEE C37.118.1a-2014 [5]. En adelante, por conveniencia, nos referiremos a estos estándares simplemente como el Std.

\section{B. Definición de Sincrofasor, Frecuencia y ROCOF}

La representación fasorial de señales senoidales es muy común en el análisis de circuitos electrónicos y sistemas eléctricos. Sea

$$
x(t)=a \cos \left(2 \pi f_{0} t+\phi\right),
$$


una onda senoidal, donde $f_{0}$ es la frecuencia nominal, $a$ es la amplitud y $\phi$ la fase. Su representación fasorial ${ }^{1}$ se define como

$$
X=a e^{j \phi} .
$$

Cuando el origen temporal $t=0$ coincide exactamente con el paso de un segundo en el estándar de tiempo UTC, $X$ se denomina representación sincrofasorial de $x(t)$. En este caso, $\phi$ representa la diferencia de fase con respecto a un coseno de frecuencia $f_{0}$ sincronizado con el tiempo UTC. En forma más general, la amplitud y la fase dependen del tiempo: $a=a(t), \phi=\phi(t)$. En este caso, la señal de tensión o corriente se puede escribir como

$$
x(t)=a(t) \cos \left[2 \pi f_{0} t+\phi(t)\right],
$$

y su sincrofasor es

$$
X(t)=a(t) e^{j \phi(t)} .
$$

Entonces, en general, los sincrofasores varían en el tiempo. La frecuencia y la ROCOF instantáneas (con respecto a los valores nominales $f_{0}$ y 0 , respectivamente) se definen a partir de la primera y segunda derivadas de la fase:

$$
\begin{gathered}
f(t)=f_{0}+\frac{1}{2 \pi} \frac{d \phi(t)}{d t}, \\
\operatorname{ROCOF}(t)=\frac{1}{2 \pi} \frac{d^{2} \phi(t)}{d t^{2}} .
\end{gathered}
$$

\section{Definición de Métricas de Desempeño}

El Std. define tres métricas de desempeño que se utilizan para evaluar todos los tests ${ }^{2}$ : error vectorial total (TVE), error en frecuencia (FE) y error en tasa de variación de frecuencia (RFE). El TVE se define del siguiente modo:

$$
\operatorname{TVE}(t)=\frac{|\widehat{X}(t)-X(t)|}{|X(t)|}
$$

donde $X(t)$ es el sincrofasor en el tiempo $t$ y $\widehat{X}(t)$ es su estimación para dicho instante de tiempo. Es decir, no es otra cosa que el módulo del error relativo. Gráficamente, se puede interpretar como la distancia entre $X(t)$ y $\widehat{X}(t)$ normalizada por $|X(t)|$. Generalmente, para estar en norma el fasor estimado debe ser tal que el TVE sea menor a algún valor, por ejemplo, el $1 \%$. Notar que cuanto mayor es el error en amplitud, menor es la tolerancia en el error de fase y viceversa. El FE se define como el error absoluto entre la frecuencia instantánea $f(t)$ de la señal y su estimación $\widehat{f}(t)$ :

$$
\mathrm{FE}(t)=|f(t)-\widehat{f}(t)| .
$$

El RFE se define como el error absoluto entre la ROCOF instantánea $f^{\prime}(t)$ de la señal y su estimación $\widehat{f}^{\prime}(t)$ :

$$
\operatorname{RFE}(t)=\left|f^{\prime}(t)-\widehat{f}^{\prime}(t)\right|
$$

\footnotetext{
${ }^{1}$ Muchas veces se trabaja con el fasor RMS definido como $X_{\mathrm{RMS}}=$ $X / \sqrt{2}$. Sin embargo, aquí preferimos por simplicidad trabajar con el fasor $X$. Obviamente siempre se puede obtener una representación a partir de la otra con un simple cambio de escala.

${ }^{2} \mathrm{La}$ excepción es el test de saltos en amplitud y fase, donde se definen otras métricas de desempeño para evaluar las respuestas transitorias. Ellas son: el sobrepico, los tiempos de respuesta del TVE, FE y RFE, y el tiempo de retardo.
}

\section{Clases de PMUs}

El Std. define dos clases de desempeño para el PMU: clase M y clase P. La clase M, o de Monitoreo, es la clase más adecuada para aplicaciones que requieren mediciones de alta exactitud y admiten una latencia considerable en el reporte de las mediciones. En cambio, la clase P, o de Protección, se utiliza en aplicaciones que exigen respuestas rápidas pero de menor exactitud. Como es de esperarse, los requisitos de respuesta rápida y alta exactitud constituyen uno de los compromisos más importantes en esta aplicación.

\section{E. Reporte de Estimaciones}

El PMU reporta los datos a un concentrador de datos para su posterior uso con una tasa dada. Cabe notar que dicha tasa no está relacionada con la tasa de muestreo del conversor AD dentro del PMU, sino que de acuerdo al Std. debe ser igual a un submúltiplo de la frecuencia nominal del sistema. Para $f_{0}=50 \mathrm{~Hz}$, se exige que la tasa de reporte $F_{s}$ pueda tomar los valores 10,25 y 50 reportes por segundo (fps). Cada tasa de reporte representa un modo de funcionamiento diferente del dispositivo pues tiene distintos requisitos.

\section{F. Consideraciones de Latencia}

La latencia del PMU se define como la diferencia temporal entre el instante en que los datos se encuentran disponibles a la salida del PMU y el instante exacto del tiempo de reporte indicado por la etiqueta temporal. Los límites máximos dependen principalmente de la tasa de reporte y la clase del PMU, ya que estos factores determinan el tipo de filtrado que se realiza. Concretamente, los límites de latencia son $2 / F_{s}$ y $7 / F_{s}$ para el desempeño clase $\mathrm{P}$ y clase $\mathrm{M}$ respectivamente.

\section{G. Motivación y contribución del artículo}

Los métodos más importantes de estimación de sincrofasores, basados en modelos de fasor constante o polinómico, tienen claras limitaciones impuestas por los mismos modelos. Luego de disctuir esto, presentamos un enfoque muy general introducido recientemente basado en técnicas de optimización convexa semi-infinita ${ }^{3}$ y mostramos la conexión que tiene con los métodos previos. Cabe mencionar que existen otros artículos que presentan una comparación de distintos métodos [6]-[8]. La contribución principal de este artículo es ampliar la perspectiva del estado del arte de la estimación de sincrofasores y enriquecerla con nuestras contribuciones en el área.

\section{H. Organización del artículo}

El resto del artículo está organizado del siguiente modo. En la Sección II presentamos algunas consideraciones generales, comunes a todos los algoritmos. Luego, en la Sección III discutimos en detalle los métodos basados en la DFT, y luego mostramos sus limitaciones. El mismo análisis se realiza para los métodos basados en la TFT en la Sección IV. Posteriormente, en la Sección V se presentan los métodos de diseño de filtros basados en CSIP, así como su relación con los métodos previos, mostrando la generalidad del enfoque de optimización. Finalmente, concluimos el artículo en la Sección VI.

${ }^{3}$ Puede pensarse que el método basado en optimización se construye a partir de una familia infinita de modelos, como veremos en la Sección V. 


\section{PRELIMINARES}

\section{A. Preprocesamiento}

Todos los algoritmos que vamos a analizar en este trabajo parten de mediciones que se tienen de una señal trifásica de tensión o corriente, que es digitalizada mediante un conversor $\mathrm{AD}$, produciendo una señal vectorial que denotaremos $\mathbf{x}_{a b c}[n]=\left[x_{a}[n], x_{b}[n], x_{c}[n]\right]^{T}$, donde $a, b, c$ representan cada una de las fases del sistema eléctrico, y que se puede descomponer del siguiente modo

$$
\mathbf{x}_{a b c}[n]=\mathbf{s}_{a b c}[n]+\mathbf{n}_{a b c}[n],
$$

donde $\mathbf{s}_{a b c}[n]$ es la componente fundamental de interés y $\mathbf{n}_{a b c}[n]$ representa interferencias (armónicas e interarmónicas) y ruido. En general, $\mathbf{s}_{a b c}[n]$ puede representarse como la suma de las componentes simétricas instantáneas [9], tal como se muestra en la Ec. (11) (ver parte inferior de la siguiente página), donde $a_{i}[n] \equiv a_{i}(n T)$ y $\phi_{i}[n] \equiv \phi_{i}(n T)$, con $i=a, b, c, 0,1,2$, son la amplitud y fase instantáneas de cada fase $(a, b, c)$ o componente $(0,1$ o positiva, 2 o negativa) correspondiente, $\omega_{0}$ es la frecuencia angular nominal del sistema de potencia, $T=2 \pi /\left(C \omega_{0}\right)$ es el período de muestreo, siendo $C$ la cantidad de muestras que se obtienen por ciclo nominal, y $\nu_{0}=\omega_{0} T=2 \pi / C$.

Sin embargo, los algoritmos no operan directamente con la señal $\mathbf{x}_{a b c}[n]$, sino que primero la transforman con el fin de construir mediciones directas del fasor de secuencia positiva de la componente fundamental, que posteriormente serán procesadas para obtener las estimaciones del sincrofasor, la frecuencia y la ROCOF. Como esta etapa es general, independiente de la elección del algoritmo de estimación, se presenta en esta sección. Dicha conversión se basa en las transformaciones ${ }^{4}$ de Clarke (o transformación $\alpha \beta \gamma$ ) y de Park (o transformación $a b c-d q o$ ), que son transformaciones de coordenadas frecuentemente utilizadas en el análisis de sistemas de potencia trifásicos [10]. Ambas son transformaciones lineales. La transformación de Clarke queda definida por la matriz constante

$$
\boldsymbol{C}=\frac{2}{3}\left[\begin{array}{ccc}
1 & -\frac{1}{2} & -\frac{1}{2} \\
0 & \frac{\sqrt{3}}{2} & -\frac{\sqrt{3}}{2}
\end{array}\right],
$$

mientras que la transformación de Park se puede representar por la siguiente matriz variante en el tiempo

$\boldsymbol{P}[n]=\frac{2}{3}\left[\begin{array}{rrr}\cos \left(\phi_{a, 0}[n]\right) & \cos \left(\phi_{b, 0}[n]\right) & \cos \left(\phi_{c, 0}[n]\right) \\ -\sin \left(\phi_{a, 0}[n]\right) & -\sin \left(\phi_{b, 0}[n]\right) & -\sin \left(\phi_{c, 0}[n]\right)\end{array}\right]$,

donde $\phi_{a, 0}[n]=\nu_{0} n, \phi_{b, 0}[n]=\nu_{0} n-2 \pi / 3$ y $\phi_{c, 0}[n]=$ $\nu_{0} n+2 \pi / 3$. Operando, obtenemos que

$$
\begin{aligned}
\mathbf{x}_{d q}[n] & =\left[\begin{array}{l}
x_{d}[n] \\
x_{q}[n]
\end{array}\right]=\boldsymbol{C} \mathbf{x}_{a b c}[n]=a_{1}[n]\left[\begin{array}{l}
\cos \left(\nu_{0} n+\phi_{1}[n]\right) \\
\sin \left(\nu_{0} n+\phi_{1}[n]\right)
\end{array}\right] \\
& +a_{2}[n]\left[\begin{array}{l}
\cos \left(-\nu_{0} n-\phi_{2}[n]\right) \\
\sin \left(-\nu_{0} n-\phi_{2}[n]\right)
\end{array}\right]+\boldsymbol{C} \mathbf{n}_{a b c}[n]
\end{aligned}
$$

\footnotetext{
${ }^{4}$ Estrictamente, usamos las transformaciones de Clark y Park reducidas pues tanto la componente $\gamma$ como la componente cero $(o)$ no contienen información del sincrofasor y, por lo tanto, no son calculadas.
}

$$
\begin{aligned}
\mathbf{y}_{d q}[n] & =\left[\begin{array}{l}
y_{d}[n] \\
y_{q}[n]
\end{array}\right]=\boldsymbol{P}[n] \mathbf{x}_{a b c}[n]=a_{1}[n]\left[\begin{array}{l}
\cos \left(\phi_{1}[n]\right) \\
\sin \left(\phi_{1}[n]\right)
\end{array}\right] \\
& +a_{2}[n]\left[\begin{array}{l}
\cos \left(-2 \nu_{0} n-\phi_{2}[n]\right) \\
\sin \left(-2 \nu_{0} n-\phi_{2}[n]\right)
\end{array}\right]+\boldsymbol{P}[n] \mathbf{n}_{a b c}[n] .
\end{aligned}
$$

Es fácil ver que hay una simple relación entre ambas transformaciones, a saber, $\boldsymbol{P}[n]=\boldsymbol{R}\left(-\nu_{0} n\right) \boldsymbol{C}$, donde $\boldsymbol{R}\left(-\nu_{0} n\right)$ es una matriz de rotación en un ángulo $-\nu_{0} n$ en sentido antihorario. En general, los algoritmos se formulan a partir de la señal $x_{d}[n]+j x_{q}[n]$, pero equivalentemente se pueden formular a partir de la señal $y_{d}[n]+j y_{q}[n]$, lo cual muchas veces ofrece una versión simplificada del método en cuestión y revela más claramente la esencia del mismo.

Observando la ecuación (15), es claro que el primer término se puede identificar como el sincrofasor $X[n]$, mientras que los otros términos representan el efecto de la componente negativa, interferencias y ruido. Cabe destacar algunas ventajas de esta transformación [11]:

1) A diferencia de una demodulación monofásica, no genera un término de doble frecuencia para señales de entrada balanceadas, es decir, con $a_{0}[n]=0 \mathrm{y}$ $a_{2}[n]=0$.

2) Filtra por completo la componente cero que, como mencionamos previamente, no contiene información útil para nuestro problema.

3) La frecuencia de la componente positiva es mapeada a $0 \mathrm{rad} . /$ muestra, mientras que la componente negativa es trasladada a $-2 \nu_{0}$ rad./muestra. Este desacoplamiento en frecuencia tiene el efecto de producir una mayor robustez ante desbalanceos de la señal de entrada.

\section{B. Algoritmos de procesamiento por bloques}

Los métodos basados en procesamiento por bloques parten de un conjunto finito de datos a partir del cual se deben estimar los parámetros de interés. Concretamente, la formulación general es la siguiente. A partir de $N$ muestras de una señal de tensión o corriente se pretende estimar mediante alguna operación matemática el fasor, la frecuencia, y la ROCOF correspondientes al tiempo de reporte. Por simplicidad, supondremos que el número de muestras en un bloque es impar. Entonces, denotaremos por $\mathbf{x}=\left\{x_{n}\right\}_{n \in \mathcal{N}}=\left[x_{-R}, \ldots, x_{0}, \ldots, x_{R}\right]^{T} \in \mathbb{C}^{N}$ al vector de muestras en un bloque de la señal $x_{d}[n]+j x_{q}[n]$ y por $\mathbf{y}=\left\{y_{n}\right\}_{n \in \mathcal{N}}=\left[y_{-R}, \ldots, y_{0}, \ldots, y_{R}\right]^{T} \in \mathbb{C}^{N}$ al vector de muestras del mismo bloque de la señal $y_{d}[n]+j y_{q}[n]$, donde $R=\frac{N-1}{2}$ y $\mathcal{N}=\{-R, \ldots, R\}$. La relación entre las componentes de ambos vectores es simplemente

$$
y_{n}=x_{n} e^{-j \nu_{0} n}, \quad n \in \mathcal{N} .
$$

Además, asumimos que el tiempo de reporte de las mediciones siempre coincide con el instante de tiempo asociado a la muestra $x_{0}$ o $y_{0}$, es decir, con el centro del bloque. Este planteo es habitual en la literatura de sincrofasores, posiblemente porque es el que se propone en el mismo Std. [4]. Notar que el hecho de que el tiempo de reporte esté en el centro del bloque hace que la estimación sea no causal. Esto puede llamar la atención del lector pero no es problemático pues, como hemos mencionado en la Sección I-F, el Std. admite cierta latencia en el reporte de las mediciones de las 
PMUs. De hecho, éste es un típico problema de suavizado en procesamiento de señales. Sin embargo, es evidente que el largo del bloque queda delimitado por la latencia máxima tolerable $t_{L}$. Concretamente, para no violar esta restricción, debemos tener necesariamente que ${ }^{5}$

$$
R \leq \frac{t_{L}}{T}
$$

Recordemos que la latencia máxima tolerable depende de la clase del PMU. Luego, la restricción sobre el largo del bloque será distinta para aplicaciones de protección (clase P) y monitoreo (clase $\mathrm{M}$ ).

\section{Algoritmos BASAdos EN LA DFT}

Tradicionalmente, el algoritmo que se ha utilizado con mayor popularidad para estimar el sincrofasor se basa en una simple transformada de Fourier discreta (DFT) [12]. En particular, como únicamente se desea conocer el fasor de la componente fundamental de la señal de entrada, la operación que se realiza para obtener la estimación del sincrofasor es la siguiente:

$$
\widehat{X}=\frac{1}{N} \sum_{n \in \mathcal{N}} x_{n} e^{-j \nu_{0} n} .
$$

Si reescribimos $\widehat{X}$ en términos de la señal $\left\{y_{n}\right\}$, tenemos

$$
\widehat{X}=\frac{1}{N} \sum_{n \in \mathcal{N}} y_{n}
$$

Luego, la interpretación de este estimador fasorial es muy sencilla: $\widehat{X}$ no es otra cosa que el promedio de la señal $\left\{y_{n}\right\}$, es decir, de la señal $\left\{x_{n}\right\}$ demodulada a frecuencia nominal. Esta observación permite hacer algunas conexiones interesantes que serán útiles posteriormente:

1) El estimador $\widehat{X}$ se puede interpretar como la salida en el instante $n=0$ de un filtro promediador, es decir, cuya respuesta impulsiva es

$$
h_{n}=\frac{1}{N} \mathbb{1}\{n \in \mathcal{N}\},
$$

donde $\mathbb{1}\{\cdot\}$ representa la función indicadora. Como el filtro promediador es simplemente un filtro pasa bajos, esto sugiere que se pueden utilizar otros filtros pasa bajos, tal como se muestra en el apéndice $\mathrm{C}$ del Std. [4]. Luego, es posible utilizar técnicas de diseño de filtros para obtener nuevos estimadores fasoriales. Esta idea se discute en detalle en la Sección V.

2) En términos estadísticos, el estimador de la DFT se puede interpretar como la media muestral de la secuencia $\left\{y_{n}\right\}$. Dicho estimador es óptimo en el

\footnotetext{
${ }^{5}$ En rigor, en la práctica debemos considerar un margen de latencia para los retardos propios del cómputo de las estimaciones. Dichos retardos dependen del hardware utilizado para la implementación del algoritmo pero generalmente son despreciables frente a la latencia propia del esquema de procesamiento por bloques. Por lo tanto, su contribución a la latencia será ignorada en este trabajo.
}

siguiente sentido. Supongamos que las mediciones se pueden modelar como

$$
x_{n}=s_{n}+w_{n}=X e^{j \nu_{0} n}+u_{n}, \quad n \in \mathcal{N},
$$

donde $X=A e^{j \phi}$ es el sincrofasor, $\left\{s_{n}\right\}$ es la señal y $\left\{u_{n}\right\}$ es ruido blanco complejo circular Gaussiano de varianza $\sigma^{2}$. Las muestras de $\left\{y_{n}\right\}$ son

$$
y_{n}=X+v_{n}, \quad n \in \mathcal{N},
$$

donde $v_{n}=e^{-j \nu_{0} n} u_{n}$. Por definición, $\left\{v_{n}\right\}$ también es ruido blanco complejo circular Gaussiano. En forma vectorial, el modelo se puede escribir como

$$
\mathbf{y}=X \mathbf{1}+\mathbf{v}
$$

donde $X$ es simplemente un parámetro complejo que se desea estimar y 1 es un vector de $N$ unos. Luego, $\mathbf{y} \sim \mathcal{C N}\left(X \mathbf{1}, \sigma^{2} \boldsymbol{I}\right)$. Es bien sabido en teoría de estimación que el estimador

$$
\widehat{X}=\left(\mathbf{1}^{T} \mathbf{1}\right)^{-1} \mathbf{1}^{T} \mathbf{y}=\frac{1}{N} \sum_{n \in \mathcal{N}} y_{n},
$$

es eficiente, es decir, es un estimador insesgado que satisface la cota de Cramér-Rao y, por lo tanto, es un estimador insesgado de mínima varianza [13]. Su varianza es entonces

$$
\operatorname{Var}(\widehat{X})=F^{-1}(X)=\frac{\sigma^{2}}{N},
$$

donde $F(X)$ es la información de Fisher de $X$ en $\mathbf{y}$.

3) Es interesante observar que es posible relacionar ambas interpretaciones anteriores mediante el concepto de filtro adaptado, que es el filtro que maximiza la relación señal a ruido a su salida y actúa como correlador de la señal y y la señal constante 1. Este concepto es clásico en procesamiento de señales [14].

Una propiedad bien conocida de la DFT es su excelente rechazo de la componente continua y las armónicas en condiciones de frecuencia nominal, que se puede entender fácilmente a partir de la ortogonalidad de la familia de señales $\left\{\left\{e^{j h \nu_{0} n}\right\}: h=0, \ldots, N-1\right\}$ [15] suponiendo que $K=N / C \in \mathbb{N}$, siendo $K$ la cantidad de ciclos nominales dentro del bloque. Concretamente, supongamos que la señal es de la forma

$$
s_{n}=X e^{j \nu_{0} n}+X_{h} e^{j h \nu_{0} n}, \quad n \in \mathcal{N},
$$

donde $h \in\{0,2, \ldots, N-1\}$ es el índice de armónica y $X_{h}$ es el fasor de la armónica. Ignorando el ruido ${ }^{6}$, tenemos entonces que

$$
\widehat{X}=X+X_{h} \frac{1}{N} \sum_{n \in \mathcal{N}} e^{j(h-1) \nu_{0} n}=X,
$$

${ }^{6}$ En lo que sigue, salvo cuando se discuta el comportamiento estadístico de un estimador, siempre ignoraremos el efecto del ruido. $\mathbf{s}_{a b c}[n]=\left[\begin{array}{l}a_{a}[n] \cos \left(\nu_{0} n+\phi_{a}[n]\right) \\ a_{b}[n] \cos \left(\nu_{0} n+\phi_{b}[n]\right) \\ a_{c}[n] \cos \left(\nu_{0} n+\phi_{c}[n]\right)\end{array}\right]=a_{0}[n]\left[\begin{array}{c}\cos \left(\nu_{0} n+\phi_{0}[n]\right) \\ \cos \left(\nu_{0} n+\phi_{0}[n]\right) \\ \cos \left(\nu_{0} n+\phi_{0}[n]\right)\end{array}\right]+a_{1}[n]\left[\begin{array}{c}\cos \left(\nu_{0} n+\phi_{1}[n]\right) \\ \cos \left(\nu_{0} n+\phi_{1}[n]-\frac{2 \pi}{3}\right) \\ \cos \left(\nu_{0} n+\phi_{1}[n]+\frac{2 \pi}{3}\right)\end{array}\right]+a_{2}[n]\left[\begin{array}{c}\cos \left(\nu_{0} n+\phi_{2}[n]\right) \\ \cos \left(\nu_{0} n+\phi_{2}[n]+\frac{2 \pi}{3}\right) \\ \cos \left(\nu_{0} n+\phi_{2}[n]-\frac{2 \pi}{3}\right)\end{array}\right]$, 
pues $\sum_{n \in \mathcal{N}} e^{j(h-1) \nu_{0} n}=\left\langle e^{j \nu_{0} n}, e^{j h \nu_{0} n}\right\rangle=0$, donde $\langle\cdot, \cdot\rangle$ es el producto interno canónico en $\mathbb{C}^{N}$.

A pesar de ser un estimador muy interesante por todas las propiedades que hemos enunciado previamente, el estimador de la DFT tiene un comportamiento pobre en condiciones de frecuencia no nominal [16], lo cual muestra que no es robusto ante pequeñas discrepancias entre el modelo de señal y la señal real. Para comprender este problema, supongamos ahora que la señal $s_{n}$ no tiene frecuencia nominal sino que existe una pequeña desviación $\delta$ de modo que

$$
s_{n}=X e^{j\left(\nu_{0}+\delta\right) n}, \quad n \in \mathcal{N} .
$$

Entonces, tendremos

$$
\widehat{X}=\frac{D_{R}(\delta)}{N} X
$$

donde $D_{R}(\delta)$ es el kernel de Dirichlet que se puede expresar como

$$
D_{R}(\delta)=\sum_{n \in \mathcal{N}} e^{j \delta n}=\frac{\sin \left(\frac{N \delta}{2}\right)}{\sin \left(\frac{\delta}{2}\right)} .
$$

Luego, vemos que el estimador del sincrofasor para frecuencias no nominales se ve afectado por la ganancia $D_{R}(\delta) / N$. Entonces, podemos observar que el TVE resulta

$$
\mathrm{TVE}=\frac{|\widehat{X}-X|}{|X|}=\left|\frac{D_{R}(\delta)}{N}-1\right| .
$$

Mediante una expansión en serie de Taylor alrededor de $\delta=0$, es fácil ver que

$$
\frac{D_{R}(\delta)}{N}-1=\frac{1-N^{2}}{24} \delta^{2}+O\left(N^{4} \delta^{4}\right),
$$

de modo que el TVE crece aproximadamente en forma cuadrática con el corrimiento en frecuencia $\delta$ y el largo del bloque $N$. Expresiones análogas para una señal monofásica se pueden encontrar en [16]. Se observa que el comportamiento de la DFT es aún peor en dicho caso por la presencia de la componente imagen. Del mismo modo, podemos observar que el rechazo de armónicas también sufre en condiciones de frecuencia no nominal pues la ortogonalidad de las exponenciales complejas deja de ser válida.

En la Fig. 1 presentamos un gráfico del TVE en función de $\Delta f=\delta /(2 \pi T)$ para bloques de distinto largo, marcando el límite del $\% 1$ establecido en el Std. considerando $T=1$ ms. También se muestran los puntos $\Delta f= \pm 2 \mathrm{~Hz}$ que se corresponden con los límites de frecuencia no nominal para el PMU clase P. Se puede observar que en todos los casos el TVE supera la cota del Std. cuando se considera el rango de frecuencias $[45,55] \mathrm{Hz}$, que se corresponde con el PMU clase M. Solamente para el bloque más corto (de un ciclo de largo), el TVE satisface la norma para el rango de frecuencias del PMU clase P.

Una generalización natural de la DFT para reducir estos efectos de leakage, es utilizar una DFT ventaneada o WDFT. En dicho caso, el estimador se puede escribir como

$$
\widehat{X}=\frac{\sum_{n \in \mathcal{N}} w_{n} y_{n}}{\sum_{n \in \mathcal{N}} w_{n}},
$$

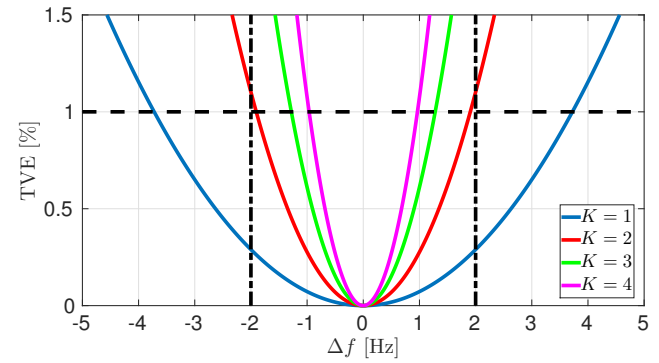

Fig. 1: TVE del estimador fasorial basado en la DFT en función del corrimiento en frecuencia $\Delta f$ para bloques de distinto largo.

donde $\left\{w_{n}\right\}$ son los coeficientes de la ventana elegida. Es claro que con esta generalización, $\widehat{X}$ es simplemente un promedio ponderado de la señal $\left\{y_{n}\right\}$. El estudio de la influencia de ventanas en aplicaciones de estimación espectral se ha estudiado ampliamente [17]-[19]. En particular, una clase rica de ventanas que se ha propuesto utilizar en la aplicación de sincrofasores es la clase cosenoidal [20], que incluye a las ventanas tradicionales de Hanning, Hamming, Blackman y Nuttall. Otra ventana atractiva, por ser una aproximación de la ventana óptima ${ }^{7}$ de Slepian [15], es la ventana de Kaiser, que se define como

$$
w_{n}=\frac{I_{0}\left(\beta \sqrt{1-\left(\frac{n}{R}\right)^{2}}\right)}{I_{0}(\beta)}, \quad n \in \mathcal{N},
$$

donde $I_{0}(\cdot)$ es la función de Bessel modificada de orden cero y $\beta$ es un parámetro no negativo que controla la forma de la ventana. Básicamente, a medida que $\beta$ aumenta, el ancho del lóbulo principal de la ventana aumenta y el área de los lóbulos secundarios disminuye. El estimador WDFT en condiciones de frecuencia no nominal será

$$
\widehat{X}=\frac{\sum_{n \in \mathcal{N}} w_{n} X e^{j \delta n}}{\sum_{n \in \mathcal{N}} w_{n}}=X \frac{W(-\delta)}{W(0)},
$$

donde $W(\nu)=\sum_{n \in \mathcal{N}} w_{n} e^{-j \nu n}$ es la transformada de Fourier (DTFT) de $\left\{w_{n}\right\}$. Luego, si la ventana satisface la condición

$$
\frac{W(-\delta)}{W(0)} \leq \frac{D_{R}(\delta)}{N}
$$

para un rango apropiado de $\delta$, se tendrá un mejor comportamiento que con el estimador DFT.

Otro algoritmo muy popular para compensar los errores que aparecen en condiciones de frecuencia no nominal es el algoritmo IpDFT o DFT interpolada [21]. La motivación del algoritmo es muy sencilla. De la Ec. (35) podemos apreciar que si conociéramos el corrimiento en frecuencia $\delta$, podríamos compensar el factor que aparece en el estimador WDFT perfectamente. En el algoritmo IpDFT, se comienza por evaluar la WDFT de la secuencia $\left\{y_{n}\right\}$ en la frecuencia nominal y en los bins adyacentes:

$$
Z(0)=X W(-\delta), \quad Z( \pm \varepsilon)=X W( \pm \varepsilon-\delta),
$$

${ }^{7}$ En el sentido de maximizar la relación energía del lóbulo principal / energía total de la ventana. 
donde $Z(\nu)$ es la DTFT de la secuencia $\left\{z_{n}\right\}$, con $z_{n}=$ $w_{n} y_{n}$ para todo $n \in \mathcal{N}$, y $\varepsilon=2 \pi / N$. Luego, se comparan los valores de $|Z(\varepsilon)|$ y $|Z(-\varepsilon)|$. Si $|Z(\varepsilon)|>|Z(-\varepsilon)|$, tendremos que $\delta>0$, y en caso contrario $\delta<0$. Esto asume que el corrimiento en frecuencia satisface $|\delta|<\varepsilon / 2$ [18], lo cual en general es cierto para la aplicación de sincrofasores, pero puede no valer para bloques muy largos. Supongamos, por ejemplo, que se satisface la condición $|Z(\varepsilon)|>|Z(-\varepsilon)|$. Luego, el siguiente paso es evaluar

$$
\gamma \doteq \frac{|Z(\varepsilon)|}{|Z(0)|}=\frac{W(-\delta+\varepsilon)}{W(-\delta)}=f(\delta),
$$

donde $f(\cdot)$ es una función que depende de la ventana elegida. Cabe notar que la igualdad $\gamma=f(\delta)$ es válida únicamente para el caso ideal en que no hay ruido ni interferencia. Finalmente, se busca invertir la relación anterior para obtener una estimación del corrimiento en frecuencia

$$
\widehat{\delta}=f^{-1}(\gamma),
$$

que luego se utilizará para compensar el estimador WDFT de la Ec. (35). Desde un punto de vista práctico, aún si $f(\cdot)$ es una función muy compleja para invertir analíticamente, una aproximación polinómica de $f^{-1}(\cdot)$ puede ser suficientemente exacta [22]. Cabe notar que por medio del algoritmo IpDFT, obtenemos además de la corrección del leakage, un estimador de frecuencia. Alternativamente, para los estimadores DFT y WDFT, se puede utilizar el estimador que se propone en el Std. basado en diferencias finitas [5]. Del mismo modo, ninguno de los métodos basados en la DFT proporciona un estimador de ROCOF, de modo que es razonable utilizar nuevamente el método propuesto en el Std. Cabe notar que dichos métodos requieren la estimación de dos sincrofasores adicionales ${ }^{8}$ por cada tiempo de reporte que no son reportados pero son necesarios para obtener estimaciones de frecuencia y ROCOF suficientemente exactas. Además, como bien se señala en el Std., los estimadores propuestos son muy sensibles al ruido.

\section{Algoritmos BASAdos EN EL MODELO DE TAYLOR-FOURIER}

Una limitación fundamental de los métodos basados en la DFT es que asumen, al menos en forma implícita, un modelo estacionario. Por lo tanto, su desempeño en general es pobre en condiciones dinámicas, es decir, cuando el sincrofasor varía en el tiempo significativamente dentro del bloque de datos [8]. En este sentido, una contribución muy importante en el área de estimación de sincrofasores fue el reconocimiento de la gran ventaja de usar un modelo de fasor dinámico [23]. Concretamente, en dicho trabajo se plantea un modelo polinómico para el sincrofasor, es decir, la señal $\left\{x_{n}\right\}$ toma la siguiente forma

$$
x_{n}=\sum_{l=0}^{L} X_{l} n^{l} e^{j \nu_{0} n}+u_{n}, \quad n \in \mathcal{N},
$$

donde $X_{0}, \ldots, X_{L} \in \mathbb{C}$ son los parámetros del modelo. El estimador que presentamos a continuación se conoce

\footnotetext{
${ }^{8} \mathrm{Si}$ el sincrofasor que se desea estimar es $X[n]$, la implementación de los estimadores de frecuencia y ROCOF por diferencias finitas requiere la estimación de $X[n-1]$ y $X[n+1]$.
}

como estimador de Taylor-Fourier (TFT) pues se basa en una expansión en Taylor del fasor dinámico referido a la frecuencia fundamental $\nu_{0}$. En términos de la señal $\left\{y_{n}\right\}$, tenemos que

$$
y_{n}=\sum_{l=0}^{L} X_{l} n^{l}+v_{n}, \quad n \in \mathcal{N} .
$$

En forma vectorial podemos escribir entonces

$$
\mathbf{y}=\mathbf{N X}+\mathbf{v},
$$

donde hemos definido la matriz

$$
\boldsymbol{N}=\left[\begin{array}{ccccc}
1 & -R & (-R)^{2} & \cdots & (-R)^{L} \\
1 & -R+1 & (-R+1)^{2} & \cdots & (-R+1)^{L} \\
\vdots & \vdots & \vdots & \vdots & \vdots \\
1 & R & R^{2} & \cdots & R^{L}
\end{array}\right]
$$

y el vector de parámetros $\mathbf{X}=\left[X_{0}, \ldots X_{L}\right]^{T}$. Notar que el modelo nuevamente es lineal en los parámetros y, además, $\mathbf{y} \sim \mathcal{C N}\left(\boldsymbol{N X}, \sigma^{2} \boldsymbol{I}\right)$. Luego, el estimador de mínimos cuadrados [23] es eficiente [13]:

$$
\widehat{\mathbf{X}}=\left(\boldsymbol{N}^{T} \boldsymbol{N}\right)^{-1} \boldsymbol{N}^{T} \mathbf{y}=\boldsymbol{G} \mathbf{y},
$$

donde $\boldsymbol{G}=\left(\boldsymbol{N}^{T} \boldsymbol{N}\right)^{-1} \boldsymbol{N}^{T}$. Por lo tanto, su matriz de covarianza es

$$
\operatorname{Cov}(\widehat{\mathbf{X}})=\boldsymbol{F}^{-1}(\mathbf{X})=\sigma^{2} \boldsymbol{G} \boldsymbol{G}^{T}=\sigma^{2}\left(\boldsymbol{N}^{T} \boldsymbol{N}\right)^{-1},
$$

donde $\boldsymbol{F}(\mathbf{X})$ es la matriz de información de Fisher de $\mathbf{X}$ en $\mathbf{y}$. Un resultado fundamental de teoría de estimación establece que la varianza del estimador de un parámetro no puede disminuir, y en general crece, cuando se estima junto con otros parámetros [13]. En nuestro caso, esto significa que la varianza del estimador TFT del sincrofasor (el parámetro $X_{0}$ ) es mayor que la varianza del estimador DFT (el parámetro $X$ ). Del mismo modo, cuanto mayor sea $L$ mejor será la aproximación polinómica del sincrofasor en general pero mayor será la varianza por el hecho de que el modelo tiene más parámetros. Esto es simplemente una manifestación de la relación de compromiso que existe entre el sesgo y la varianza de un estimador. Concretamente, el sesgo decrece con $L$ pero la varianza crece con $L$. El caso $L=0$, que se corresponde con el estimador DFT clásico da el estimador de mínima varianza pero con un sesgo máximo para un fasor dinámico. Podemos decir que, en dicho caso, el modelo es muy simple o existe underfitting. Por el contrario, cuando se utiliza $L>0$, se obtiene una mejor aproximación al fasor dinámico a expensas de un incremento en la varianza del estimador. Eventualmente, con $L$ suficientemente grande, la aproximación será excelente, pero a expensas de un incremento considerable en la varianza. En este caso, el modelo es demasiado complejo o existe overfitting. Además, siempre se debe tener $L \ll N$ para evitar que la matriz $G$ esté mal condicionada [24].

Es interesante observar que a partir de la estimación de los parámetros $X_{0}, X_{1}$ y $X_{2}$ se pueden obtener directamente estimaciones de la frecuencia angular $\omega$ y la ROCOF angular $\alpha$, también en el centro del bloque de datos, explotando las 
relaciones entre las derivadas de un fasor dinámico y su frecuencia y ROCOF [23], [25]. Las relaciones son:

$$
\begin{aligned}
& \widehat{\omega}=\frac{1}{T} \operatorname{Im}\left(\frac{\widehat{X}_{1}}{\widehat{X}_{0}}\right), \\
& \widehat{\alpha}=\frac{2}{T^{2}}\left[\operatorname{Im}\left(\frac{\widehat{X}_{2}}{\widehat{X}_{0}}\right)-\operatorname{Re}\left(\frac{\widehat{X}_{1}}{\widehat{X}_{0}}\right) \operatorname{Im}\left(\frac{\widehat{X}_{1}}{\widehat{X}_{0}}\right)\right] .
\end{aligned}
$$

Una generalización directa del estimador TF, que se presenta en [24], se basa en resolver un problema de cuadrados mínimos ponderado (WLS) en lugar de un problema de LS. La motivación es análoga a la que justifica utilizar un estimador WDFT. Es decir, el estimador del vector de parámetros $\mathbf{X}$ surge de resolver

$$
\widehat{\mathbf{X}}=\left(\boldsymbol{N}^{T} \boldsymbol{W}^{T} \boldsymbol{W} \boldsymbol{N}\right)^{-1} \boldsymbol{N}^{T} \boldsymbol{W}^{T} \boldsymbol{W} \mathbf{y}=\boldsymbol{G} \mathbf{y},
$$

con $\boldsymbol{W}=\operatorname{diag}\left(\left\{w_{n}\right\}\right)$, donde $\left\{w_{n}\right\}$ son los pesos o coeficientes de la ventana elegida. Por razones obvias, el estimador de la Ec. (48) se denomina estimador WTF. Por supuesto, la ecuación (48) se reduce a la ecuación (44) cuando se toma una ventana rectangular, es decir, $\boldsymbol{W}=\boldsymbol{I}$. En [24], los autores proponen trabajar con la ventana de Kaiser, que hemos introducido en la Ec. (34).

Es interesante analizar, al igual que para el estimador basado en la DFT, cómo afecta un corrimiento en frecuencia a la estimación del sincrofasor. Nuevamente, ignoramos el ruido y partimos del modelo de señal presentado en la (28). Entonces, el problema se reduce a hallar la mejor aproximación polinómica, en el sentido LS o WLS, de una señal exponencial compleja que se puede escribir como

$$
\mathbf{y}=X \mathbf{e}(\delta)
$$

donde $\mathbf{e}(\delta)=\left[e^{-j \delta R}, \ldots, 1, \ldots, e^{j \delta R}\right]^{T}$. La solución, como ya vimos, está dada en general por la ecuación (48). Es fácil verificar que el TVE y el FE entonces están dados por

$$
\begin{aligned}
\mathrm{TVE} & =\left|\mathbf{g}_{0}^{T} \mathbf{e}(\delta)-1\right|, \\
\mathrm{FE} & =\frac{1}{2 \pi T}\left|\operatorname{Im}\left\{\frac{\mathbf{g}_{1}^{T} \mathbf{e}(\delta)}{\mathbf{g}_{0}^{T} \mathbf{e}(\delta)}\right\}-\delta\right|,
\end{aligned}
$$

donde $\mathbf{g}_{i}^{T}$ es la fila $i$-ésima de la matriz $\boldsymbol{G}$ con $i=0, \ldots, L$. Además, se puede demostrar que en este caso el RFE es nulo pues los estimadores WTFT se pueden interpretar como un banco de filtros diferenciador de fase lineal [24], [26]. En la Figs. 2 y 3 mostramos cómo afecta el corrimiento en frecuencia $\Delta f=\delta /(2 \pi T)$ a la estimación del sincrofasor y la frecuencia mediante el estimador WTFT utilizando distintas ventanas de Kaiser, es decir, variando el parámetro $\beta$. En las simulaciones se utiliza $L=3$ tal como se propone en [24]. Se puede observar que el comportamiento del estimador mejora al incrementar $\beta$, es decir, cuando el ancho de banda del espectro de la la ventana es suficientemente grande. Además, el resultado es mejor para bloques de datos cortos, lo cual es esperable por la naturaleza local de la aproximación de Taylor. Sin embargo, cabe notar que esto también significa que el estimador WTFT será más sensible al ruido y las señales de interferencia. En [24], posiblemente por esta consideración, se adopta el valor $\beta=8$ para bloques de largo $K=4$.

Es interesante preguntarse si es posible usar directamente $L=2$, pues para los parámetros que nos interesa estimar

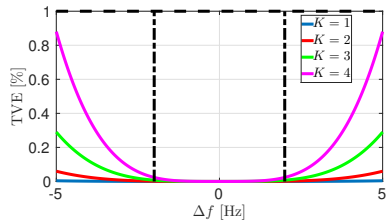

(a) $\beta=0$

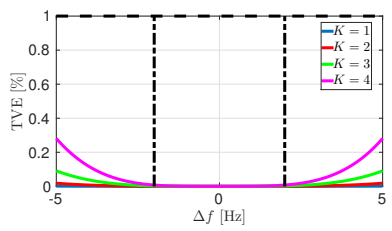

(c) $\beta=4$

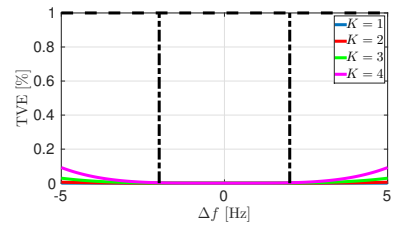

(e) $\beta=8$

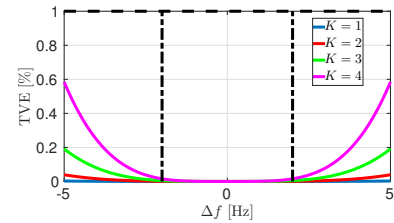

(b) $\beta=2$

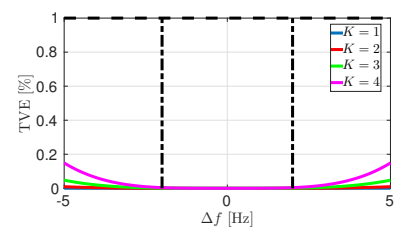

(d) $\beta=6$

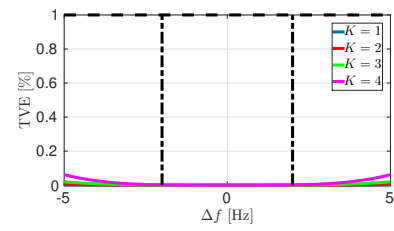

(f) $\beta=10$
Fig. 2: TVE para el estimador de Taylor-Fourier con $L=3$ para distintas ventanas de Kaiser.

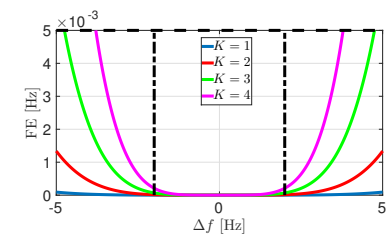

(a) $\beta=0$

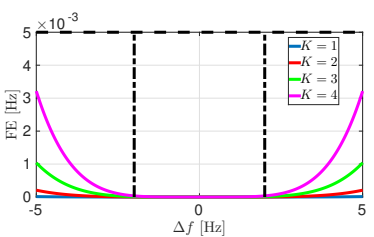

(c) $\beta=4$

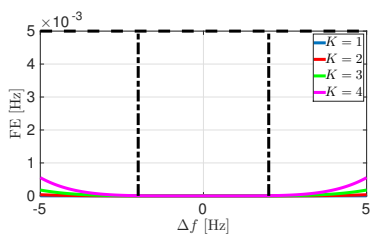

(e) $\beta=8$

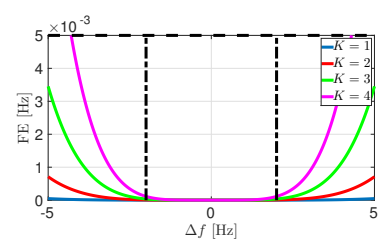

(b) $\beta=2$

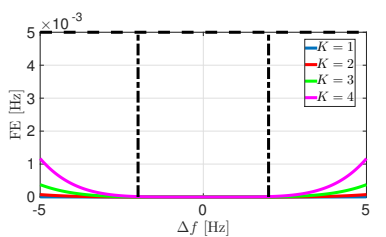

(d) $\beta=6$

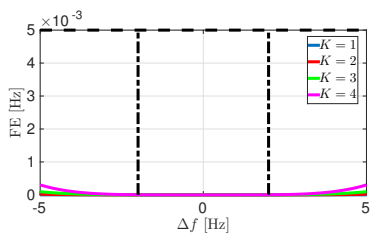

(f) $\beta=10$
Fig. 3: FE para el estimador de Taylor-Fourier con $L=3$ para distintas ventanas de Kaiser.

esto es suficiente, tal como muestran las Ecs. (46) y (47). Esta pregunta tiene sentido desde un punto de estadístico pues, como se mencionó antes, en general el agregado de parámetros genera un deterioro en la varianza del estimador. Por lo tanto, tiene sentido intentar usar el valor de $L$ mínimo compatible con nuestro problema. Desafortunadamente, como puede verse en la Fig. 4 , al utilizar $L=2$ el FE crece considerablemente y excede el límite impuesto por 
el Std., especialmente si se consideran las restricciones de un PMU clase M. Además, este comportamiento no mejora demasiado usando ventanas de banda ancha. Esto muestra que un polinomio cuadrático es un modelo demasiado simple como para describir al sincrofasor en condiciones de frecuencia no nominal, lo cual se debe simplemente al hecho de que la aproximación de mínimos cuadrados de la señal $\left\{e^{j \delta n}\right\}$ con un polinomio de grado $L=2$ no es suficientemente buena. Por lo tanto, concluimos que $L=3$ representa efectivamente una buena relación de compromiso entre el sesgo y la varianza del estimador, o bien, la exactitud y la complejidad del modelo.

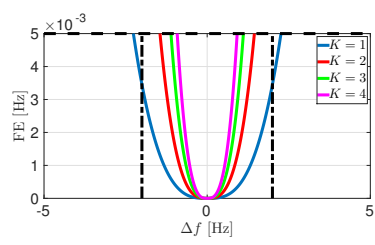

(a) $\beta=0$

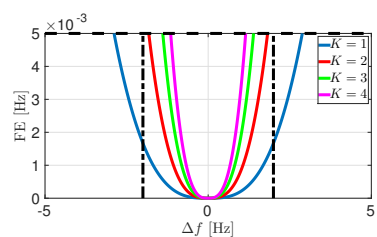

(c) $\beta=4$

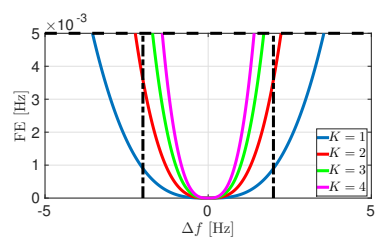

(e) $\beta=8$
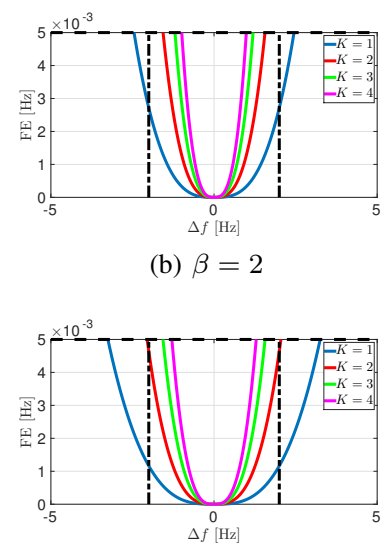

(d) $\beta=6$

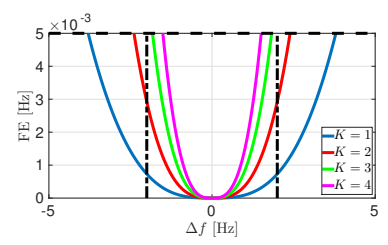

(f) $\beta=10$ (b) $\beta=2$

Fig. 4: FE para el estimador de Taylor-Fourier con $L=2$ para distintas ventanas de Kaiser.

Existen otras variaciones y extensiones interesantes del estimador TFT que presentamos brevemente a continuación. En el artículo [27] se ha propuesto referenciar el modelo de Taylor-Fourier a una frecuencia distinta a la nominal, que debe ser estimada previamente, para mejorar su comportamiento en los casos en que la frecuencia difiere notablemente de la frecuencia nominal. En particular, se propone realizar una estimación en dos etapas. En la primera etapa, se estima la frecuencia fundamental de la señal mediante el algoritmo IpDFT, descripto en la Sección III. En la segunda etapa, se estima el modelo de fasor dinámico pero referido a la frecuencia hallada en el paso anterior en lugar de la frecuencia nominal.

Otra generalización de los estimadores de Taylor-Fourier se basa en el concepto de lazos de enganche de fase (PLL) [28]. En dicho caso, se utiliza un modelo de la forma

$$
y_{n}=\sum_{l=0}^{L} X_{l} n_{l} e^{j \theta_{n}}+v_{n}, \quad n \in \mathcal{N},
$$

donde $\theta_{n}=\sum_{l=0}^{L} \eta_{l, k} n^{l}$ es un polinomio cuyos coeficientes $\eta_{l, k}$ varían con el número de bloque $k$ en forma recursiva.
Una posible estrategia de adaptación que se sugiere en [28] es utilizar los coeficientes de la fase hallados a partir de la estimación del vector $\mathbf{X}$ en el bloque $(k-1)$-ésimo como innovaciones para actualizar los coeficientes del polinomio de fase en el bloque $k$-ésimo. La ventaja de este enfoque es que permite referenciar el modelo no solamente a una frecuencia no nominal sino también a derivadas de fase superiores no nulas. Por ejemplo, para $L=2$, el método permite referenciar el modelo a una ROCOF no nula, lo cual es útil en condiciones dinámicas. Luego, la aproximación polinómica se hace solamente sobre el remanente del fasor dinámico que no se puede modelar como una exponencial compleja con fase cuadrática.

Finalmente, debemos notar que es posible extender el modelo de Taylor-Fourier para incluir componentes armónicas y, si se estiman previamente sus frecuencias mediante algún método como la IpDFT, también interarmónicas [29]. En este caso, la señal estará compuesta por $M+1$ componentes de Taylor-Fourier centradas en las frecuencias $\nu_{m}$, es decir

$$
x_{n}=\sum_{m=0}^{M} \sum_{l=0}^{L} X_{l, m} n^{l} e^{j \nu_{m} n}+u_{n}, \quad n \in \mathcal{N} .
$$

Notar que para las componentes armónicas tenemos la siguiente relación entre las frecuencias: $\nu_{m}=(m+1) \nu_{0}$. En forma vectorial, este modelo se puede escribir como $\mathbf{x}=\boldsymbol{A X}+\mathbf{u}$, donde $\boldsymbol{A}=\left[\begin{array}{llll}\boldsymbol{D}\left(\nu_{0}\right) \boldsymbol{N} & \ldots & \boldsymbol{D}\left(\nu_{M}\right) \boldsymbol{N}\end{array}\right]$. Cuando realizamos la demodulación de la frecuencia nominal $\nu_{0}$, obtenemos el modelo de la señal y como

$$
\mathbf{y}=\boldsymbol{B X}+\mathbf{v}
$$

donde

$$
\boldsymbol{B}=\boldsymbol{D}^{H}\left(\nu_{0}\right) \boldsymbol{A}=\left[\begin{array}{lll}
\boldsymbol{N} & \ldots & \boldsymbol{D}\left(\nu_{M}-\nu_{0}\right) \boldsymbol{N}
\end{array}\right] .
$$

Nuevamente podemos definir el estimador de Taylor-Fourier como la solución WLS de este problema, de modo que obtenemos

$$
\widehat{\mathbf{X}}=\left(\boldsymbol{B}^{H} \boldsymbol{B}\right)^{-1} \boldsymbol{B}^{H} \mathbf{X}=\boldsymbol{H} \mathbf{X},
$$

donde ahora el vector de parámetros es $\mathbf{X}=$ $\left[X_{0,0}, \ldots, X_{L, 0}, X_{0,1} \ldots X_{L, M}\right]^{T}$. Cabe notar que la matriz $\boldsymbol{H}$ puede precomputarse solamente para el modelo a frecuencia nominal con una cantidad prefijada de armónicas. En dicho caso, la complejidad del algoritmo crece linealmente con la dimensión del vector de parámetros, es decir, $(M+1)(L+1)$. Si bien es posible aplicar las generalizaciones del estimador TFT de una componente a este caso más general, hay que notar que el costo del algoritmo crece muy rápidamente con el número de componentes del modelo. Por ejemplo, la matriz que hay que invertir en la Ec. (56) es una matriz compleja de $(M+1)(L+1) \times(M+1)(L+1)$, de modo que la complejidad computacional $^{9}$ crece como $O\left([(M+1)(L+1)]^{3}\right)$. Además, como se discutió previamente, existen otro problemas aún más serios que ocurren cuando la cantidad de parámetros a estimar es similar a la cantidad de muestras del bloque

${ }^{9}$ Una forma habitual de resolver un sistema lineal que involucra una matriz hermitiana definida positiva es mediante la descomposición de Cholesky. 
de datos. Por un lado, cuando el modelo es demasiado complejo, tenemos un problema de overfitting. Por otro lado, para que la matriz $\boldsymbol{B}^{H} \boldsymbol{B}$ esté bien condicionada debemos tener $(M+1)(L+1) \ll N$. Esta restricción muestra que no es factible utilizar este método con bloques cortos. Una implementación más eficiente del método, que explota el hecho de que solamente se deben estimar los primeros $L+1$ parámetros de $\mathbf{X}$, y provee adaptación de la referencia de frecuencia, se presenta en [30]. Aún así, cuanto más detallado es el modelo de la señal (determinado por el grado de los polinomios de Taylor-Fourier y la cantidad de componentes del modelo), más costoso es el algoritmo.

\section{Métodos basados EN EL Diseño DE FILTROS}

Una limitación evidente de los algoritmos basados en el modelo de Taylor-Fourier es que tienen un comportamiento pobre ante cambios abruptos en la amplitud y/o fase del sincrofasor, pues la aproximación polinómica de una función con discontinuidades presenta un sobrepico fundamental por el clásico fenómeno de Gibbs [31]. Esto motiva buscar otro enfoque que no dependa de un modelo tan restrictivo para resolver el problema de estimación de sincrofasores. En este sentido, podemos decir que los métodos que se discuten en esta sección, basados en criterios de diseño de filtros, no están ligados a un modelo particular. Recientemente, se han propuesto criterios de diseño de filtros para estimación de sincrofasores, frecuencia y ROCOF basados en restricciones en el dominio de la frecuencia [32], [33], que dan por resultado máscaras espectrales. Dichas máscaras espectrales pueden utilizarse luego como base para el diseño de filtros, por ejemplo, mediante el clásico algoritmo de ParksMcClellan [15]. Sin embargo, la gran limitación de estos criterios es que no incorporan explícitamente los requisitos que se tienen en el dominio temporal, lo cual significa que la formulación del problema en ambos casos es incompleta. Una formulación completa del problema de estimación del sincrofasor y sus derivadas, lo cual permite obtener la frecuencia y la ROCOF con ecuaciones similares a las Ecs. (46) y (47), con restricciones en el dominio del tiempo y la frecuencia fue presentada en los trabajos [26], [34]. La idea básica es que el problema de los diseños de los filtros se puede expresar como un programa de optimización semiinfinita (SIP) [35]. Los SIPs son problemas de optimización que involucran una cantidad finita de variables pero una cantidad infinita de restricciones [36]. Concretamente, un problema SIP en general se puede escribir como

$$
\begin{array}{ll}
\min _{\mathbf{x}} & f(\mathbf{x}) \\
\text { subject to } & g_{k}\left(\mathbf{x}, \mathbf{y}_{k}\right) \leq 0, \quad \mathbf{y}_{k} \in Y_{k}, \quad k=1, \ldots, p,
\end{array}
$$

donde $\mathbf{x} \in \mathbb{R}^{n}$ son las variables de optimización, $Y_{k} \subset \mathbb{R}^{m_{k}}$ son conjuntos infinitos (típicamente se asume que además son compactos), $f: \mathbb{R}^{n} \rightarrow \mathbb{R}$ es la función costo, y $g_{k}$ : $\mathbb{R}^{n} \times Y_{k} \rightarrow \mathbb{R}$ son las funciones de restricción del problema. El conjunto de factibilidad del problema es

$F=\left\{\mathbf{x} \in \mathbb{R}^{n}: g_{k}\left(\mathbf{x}, \mathbf{y}_{k}\right) \leq 0\right.$ para todo $\left.\mathbf{y}_{k} \in Y_{k}, k=1, \ldots, p\right\}$
En particular, si $f$ y $g_{k}$ (para cada $\mathbf{y}_{k} \in Y_{k}$ ) son funciones convexas de $\mathbf{x}$, decimos que tenemos un programa convexo semi-infinito (CSIP) y el conjunto $F$ es convexo. Esta es la clase de problema que nos interesa y tiene la siguiente importante propiedad: un mínimo local es también un mínimo global [37]. Obviamente esto impacta fuertemente en las propiedades de convergencia de los algoritmos que resuelven problemas CSIP. Existen diversos solvers para resolver este tipo de problemas de optimización [38], [39].

En nuestro problema, usamos un banco de filtro diferenciador que consiste de tres filtros de fase lineal $H_{i}(\nu)$, $i=0,1,2$ [26], [34]. El primer filtro, $H_{0}(\nu)$, que se utiliza para obtener el estimador del sincrofasor, se elige como un filtro FIR tipo I, para producir una respuesta en amplitud par y un retardo de grupo entero. Del mismo modo, el segundo filtro, $H_{1}(\nu)$, que es un filtro diferenciador de primer orden, se elige como un filtro tipo III para producir una respuesta en amplitud impar y un retardo de grupo entero. Finalmente, el tercer filtro, $H_{2}(\nu)$, que es un diferenciador de segundo orden, nuevamente se elige como un filtro tipo I. Sin pérdida de generalidad, consideramos que los órdenes de los filtros son todos iguales a $N-1$. Las expresiones para las respuestas en amplitud son entonces

$$
A_{i}(\nu)=\mathbf{g}_{i}^{T}(\nu) \mathbf{a}_{i}, \quad i=0,1,2,
$$

donde $\mathbf{g}_{0}(\nu)=[1, \cos (\nu), \ldots, \cos (R \nu)]^{T}, \mathbf{g}_{1}(\nu)=$ $[\sin (\nu), \ldots, \sin (R \nu)]^{T}, \mathbf{g}_{2}(\nu)=\mathbf{g}_{0}(\nu)$, y nuevamente $R=$ $(N-1) / 2$. Los vectores $\mathbf{a}_{i}$ representan los coeficientes del filtro en la representación de fase lineal [15].

Claramente, en nuestro caso, las variables de optimización son los coeficientes $\mathbf{a}_{i}$ de los filtros. Como se señala en los trabajos [26], [34], si se plantea el problema del diseño de los filtros $A_{i}(\nu)$ en forma secuencial, se obtienen tres programas CSIP, uno para cada filtro. La elección de las funciones costo para los problemas que se propone en [26] es

$$
f_{i}\left(\mathbf{a}_{i}\right)=\lambda_{i}\left\|A_{i}-A_{i}^{\mathrm{id}}\right\|_{2, \Omega_{1}}^{2}+\left(1-\lambda_{i}\right)\left\|A_{i}\right\|_{2, \Omega_{2}}^{2},
$$

donde $i=0,1,2$, y $\Omega_{1}, \Omega_{2} \subseteq[-\pi, \pi]$ son conjuntos de frecuencia dados. Típicamente $\Omega_{1}$ es la unión de la banda de paso y la banda de transición de los filtros, y $\Omega_{2}$ es la banda de rechazo. Las funciones $A_{i}^{\text {id }}$ son las respuestas en amplitud ideales de cada filtro en la banda de paso, es decir, $A_{0}^{\text {id }}(\nu)=1, A_{1}^{\text {id }}(\nu)=\frac{\nu}{T}$, y $A_{2}^{\text {id }}(\nu)=-\frac{\nu^{2}}{T^{2}}$. Además, los parámetros $\lambda_{i} \in[0,1]$ se definen de acuerdo a la relación de compromiso deseada entre la distorsión de la señal de interés y la reducción de la energía de las señales de interferencia y el ruido. Notar que las funciones costo $f_{i}(\cdot)$ son funciones cuadráticas convexas en $\mathbf{a}_{i}$, de modo que se pueden escribir como $f_{i}\left(\mathbf{a}_{i}\right)=\mathbf{a}_{i}^{T} \boldsymbol{P}_{i} \mathbf{a}_{i}+\mathbf{q}_{i}^{T} \mathbf{a}_{i}+r_{i}$, donde $\boldsymbol{P}_{i}$ son matrices semidefinidas positivas. Esta familia de funciones costo es muy general. Por ejemplo, si tomamos $\lambda_{i}=0$ y $\Omega_{2}=$ $[-\pi, \pi]$, obtenemos que la función costo es proporcional a la potencia de ruido a la salida de los filtros, de modo que estaríamos minimizando la contribución del ruido al error.

Las restricciones del problema están formuladas detalladamente en el trabajo [26]. Aquí, con el fin de ilustrar el enfoque CSIP, presentaremos algunos ejemplos de la formulación de las restricciones semi-infinitas. Por ejemplo, una gran limitación del estimador clásico de la DFT consiste 
en su comportamiento pobre en condiciones de frecuencia no nominal, tal como se discutió ampliamente en la Sección III. Cuando se contempla que la señal tiene una frecuencia no nominal, tenemos el modelo de señal $y_{n}=X e^{j \delta n}$, donde $\delta$ pertenece al conjunto de frecuencias de la banda de paso $\Omega_{\mathrm{pb}}$. Si forzamos al TVE a estar acotado por una cota superior $\mathrm{TVE}_{\mathrm{STA}}$, relacionada con pero no necesariamente igual al límite del Std., obtenemos la siguiente restricción convexa semi-infinita:

$$
\mathrm{TVE}=\left|A_{0}(\delta)-1\right| \leq \mathrm{TVE}_{\mathrm{STA}}, \quad \delta \in \Omega_{\mathrm{pb}} .
$$

En forma similar, si evaluamos el FE e imponemos que sea menor a cierto valor, digamos $\mathrm{FE}_{\mathrm{STA}}$, obtenemos la restricción semi-infinita

$$
\mathrm{FE}=\frac{1}{2 \pi}\left|\frac{A_{1}(\delta)}{A_{0}^{*}(\delta)}-\frac{\delta}{T}\right| \leq \mathrm{FE}_{\mathrm{STA}}, \quad \delta \in \Omega_{\mathrm{pb}} .
$$

Cabe notar que en esta expresión, la respuesta en amplitud $A_{0}^{*}$ (parametrizada por $\mathbf{a}_{0}^{*}$ ) representa la solución del problema de optimización correspondiente al filtro $A_{0}$, que se debe resolver previamente para poder plantear el problema de optimización correspondiente al filtro $A_{1}$. La restricción anterior es del tipo convexa semi-infinita en los coeficientes $\mathbf{a}_{1}$. Por último, tal como se mencionó en la Sección IV, el RFE es exactamente nulo para este modelo de señal, por el hecho de que los filtros $H_{i}$ son de fase lineal. Por lo tanto, no es necesario imponer ninguna restricción sobre $A_{2}$ para este test.

Otra restricción que es interesante presentar es la que se obtiene cuando se consideran los tests de saltos en fase y amplitud que aparecen en el Std. [4], pues esta es la gran limitación que tienen los algoritmos basados en TFT y es una de las grandes motivaciones para utilizar el enfoque CSIP. Comenzamos considerando un salto en amplitud y luego uno en fase. El modelo de señal para un salto en amplitud es

$$
y_{n}=X[n]=X(1+\Delta a u[n]), \quad n \in \mathcal{N},
$$

donde $u[n]$ es el escalón unitario, y $\Delta a$ es el salto en amplitud. En este caso, la estimación del fasor es simplemente

$$
\widehat{X}[n]=X\left(A_{0}(0)+\Delta a s_{0}[n]\right), \quad n \in \mathcal{N},
$$

donde $s_{0}[n]$ es la respuesta al escalón del filtro $A_{0}$. Supongamos, por simplicidad, que el salto es positivo: $\Delta a>0$. El caso $\Delta a<0$ se puede tratar en forma similar. Luego, el sobrepico en amplitud (AO) en este caso se puede escribir como

$$
\begin{aligned}
\mathrm{AO} & =\max _{n=0, \ldots, R} \frac{|\widehat{X}[n]|-|X[n]|}{|X[n]|} \\
& =\max _{n=0, \ldots, R} \frac{\left(A_{0}(0)+\Delta a s_{0}[n]\right)-(1+\Delta a)}{1+\Delta a},
\end{aligned}
$$

donde $A_{0}(0)=\mathbf{g}_{0}^{T}(0) \mathbf{a}_{0} \quad$ y $s_{0}[n]=\mathbf{u}_{0}^{T}[n] \mathbf{a}_{0}$, con $\left(\mathbf{u}_{0}[n]\right)_{k_{0}}=\frac{u\left[n+k_{0}\right]+u\left[n-k_{0}\right]}{2}$ para $k_{0}=0, \ldots, R$. Luego, la restricción $\mathrm{AO} \leq \mathrm{AO}_{\max }$ se puede expresar fácilmente como $R+1$ restricciones lineales en $\mathbf{a}_{0}$, que son por supuesto compatibles con el enfoque CSIP. Del mismo modo, para un salto en fase tenemos el siguiente modelo de señal

$$
y_{n}=X[n]=X e^{j \Delta \phi u[n]}, \quad n \in \mathcal{N},
$$

donde $\Delta \phi$ es el salto en fase. En este caso, el estimador dentro del bloque de datos se puede escribir como

$$
\widehat{X}[n]=X \mathbf{v}_{0}^{H}[n] \mathbf{a}_{0}, \quad n \in \mathcal{N},
$$
donde $\quad\left(\mathbf{v}_{0}^{H}[n]\right)_{k_{0}}=\frac{e^{j \Delta \phi u\left[n+k_{0}\right]}+e^{j \Delta \phi u\left[n-k_{0}\right]}}{2}$,
$\left(\mathbf{v}_{1}^{H}[n]\right)_{k_{1}}=\frac{e^{j \Delta \phi u\left[n+k_{1}\right]}-e^{j \Delta \phi u\left[n-k_{1}\right]}}{2}, \quad\left(\mathbf{v}_{2}^{H}[n]\right)_{k_{2}}=$ $\frac{e^{j \Delta \phi u\left[n+k_{2}\right]}+e^{j \Delta \phi u\left[n-k_{2}\right]}}{2}, k_{0}, k_{2}=0, \ldots, R, k_{1}=1, \ldots, R$. Consideremos nuevamente un salto positivo, $\Delta \phi>0$, para simplificar la presentación. El sobrepico en fase (PO) entonces se puede escribir como

$\mathrm{PO}=\max _{n=0, \ldots, R} \frac{\angle \widehat{\mathcal{X}}[n]-\angle \mathcal{X}[n]}{\Delta \phi}=\max _{n=0, \ldots, R} \frac{\angle \mathbf{v}_{0}^{H}[n] \mathbf{a}_{0}-\Delta \phi}{\Delta \phi}$.

Desafortunadamente, la restricción $\mathrm{PO} \leq \mathrm{PO}_{\max }$ produce restricciones no convexas. Sin embargo, notando que $\angle \mathbf{v}_{0}^{H}[n] \mathbf{a}_{0}$ oscilará en un pequeño entorno alrededor de $\Delta \phi$ luego del salto, podemos relajar la restricción, reemplazándola por

$$
\operatorname{Im}\left\{\mathbf{v}_{0}^{H}[n]\right\} \mathbf{a}_{0} \leq \tan \left[\Delta \phi\left(1+\mathrm{PO}_{\max }\right)\right] \operatorname{Re}\left\{\mathbf{v}_{0}^{H}[n]\right\} \mathbf{a}_{0},
$$

para $n=0, \ldots, R$, que son $R+1$ restricciones lineales ordinarias en $\mathbf{a}_{0}$. Estas formulaciones permiten entonces controlar precisamente, mediante los parámetros $\mathrm{AO}_{\max } \mathrm{y}$ $\mathrm{PO}_{\text {max }}$, los sobrepicos en amplitud y fase cuando ocurren cambios abruptos en dichos parámetros, lo cual es una solución al fenómeno de Gibbs que se presenta en los estimadores basados en TF. Para demostrar esto, presentamos en la Fig. 5 la respuesta a un salto en fase de un estimador CSIP y un estimador TFT de dos ciclos. El estimador CSIP fue diseñado para satisfacer los requisitos del estándar, mientras que para el estimador TFT se utilizó una ventana rectangular (o ventana de Kaiser con $\beta=0$ ) pues con $\beta>0$ el estimador presenta un ancho de banda excesivo. La diferencia en el sobrepico de ambos estimadores es notable. De hecho, el sobrepico del estimador CSIP es despreciable (está en el orden de $10^{-10 \%}$ ), mientras que el estimador de TFT presenta un sobrepico igual a $8,02 \%$, que excede considerablemente al límite del $5 \%$ que establece el Std. para el PMU clase P. Este es un claro ejemplo de los beneficios de incluir explícitamente las restricciones del problema en la etapa de diseño.

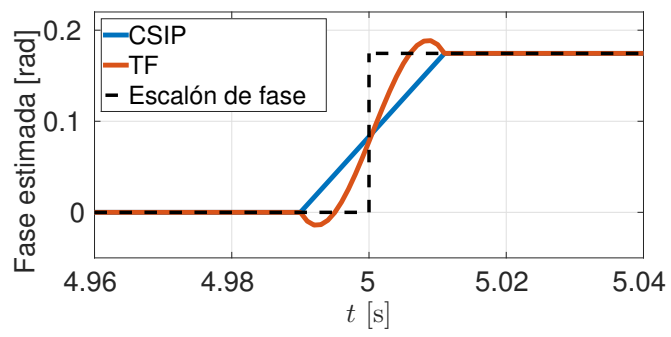

Fig. 5: Respuesta a un salto en fase de $\pi / 18$ rad. de los estimadores CSIP y TFT.

Es interesante observar que los estimadores que se obtienen con el banco de filtros diferenciador diseñado con un enfoque CSIP son generalizaciones de los estimadores DFT, WDFT, TF, y WTF. A continuación mostramos 
explícitamente esta conexión. Comencemos notando que todos los estimadores se pueden interpretar como la respuesta de filtros FIR, de modo que en general se pueden parametrizar por uno o más vectores $\mathbf{h}_{i}$. Los estimadores DFT y WDFT se pueden parametrizar por un único vector $\mathbf{h}_{0}$. Recordemos de la Sección III que el estimador DFT es un estimador lineal insesgado de mínima varianza para el modelo de fasor constante. Cuando se tiene ruido blanco, la potencia de ruido a la salida es proporcional a $\left\|\mathbf{h}_{0}\right\|^{2}$. Luego, el estimador de la DFT se puede obtener como la solución del siguiente problema de optimización:

$$
\begin{aligned}
& \min _{\mathbf{h}_{0}}\left\|\mathbf{h}_{0}\right\|^{2} \\
& \text { sujeto a } \quad H_{0}(0)=\mathbf{h}_{0}^{T} \mathbf{1}=1,
\end{aligned}
$$

como puede verificarse fácilmente [13]. Del mismo modo, el estimador WDFT se puede interpretar como un estimador lineal insesgado de mínima varianza cuando la matriz de covarianza $\boldsymbol{\Sigma}$ del ruido $\mathbf{v}$ es modelada como una matriz diagonal y donde las varianzas satisfacen ${ }^{10} \sigma_{n}^{2}=1 / w_{n}$. De este modo, la interpretación estadística de la WDFT es que las muestras que son pesadas más fuertemente son muestras de varianza pequeña y vicecersa. Luego, la WDFT es la solución del siguiente problema de optimización

$$
\begin{aligned}
& \min _{\mathbf{h}_{0}} \mathbf{h}_{0}^{T} \boldsymbol{\Sigma} \mathbf{h}_{0} \\
& \text { sujeto a } \quad H_{0}(0)=\mathbf{h}_{0}^{T} \mathbf{1}=1,
\end{aligned}
$$

donde $\boldsymbol{\Sigma}=\boldsymbol{W}^{-1}=\operatorname{diag}\left(\left\{w_{n}^{-1}\right\}\right)$. Cabe notar que en este planteo uno podría usar incluso información de correlación entre distintas muestras del ruido si se conociera o estimara previamente, lo cual generaliza la WDFT. La solución en general será ${ }^{11}$

$$
\mathbf{h}_{0}=\frac{\boldsymbol{\Sigma}^{-1} \mathbf{1}}{\mathbf{1}^{T} \boldsymbol{\Sigma}^{-1} \mathbf{1}}
$$

de modo que el estimador es

$$
\widehat{X}=\mathbf{h}_{0}^{T} \mathbf{y}=\frac{\mathbf{1}^{T} \boldsymbol{\Sigma}^{-1} \mathbf{y}}{\mathbf{1}^{T} \boldsymbol{\Sigma}^{-1} \mathbf{1}},
$$

que generaliza a la Ec. (33).

Consideremos ahora el estimador TF, que también es un estimador lineal insesgado y de mínima varianza, pero para un modelo de fasor polinómico. En este caso, el estimador queda determinado por $L+1$ filtros, de modo que la variable de optimización es una matriz $\boldsymbol{H}=\left[\mathbf{h}_{0}, \ldots, \mathbf{h}_{L}\right]$. Para que el estimador $\widehat{\mathbf{X}}=\boldsymbol{H}^{T} \mathbf{y}$ sea insesgado, debemos pedir que $\mathbb{E}[\widehat{\mathbf{X}}]=\mathbf{X}$ para cualquier $\mathbf{X} \in \mathbb{C}^{L+1}$. Entonces, obtenemos la condición

$$
\boldsymbol{H}^{T} \boldsymbol{N}=\boldsymbol{I}
$$

Por otro lado, notar que la potencia total de ruido a la salida del banco de filtros diferenciador es proporcional a $\left\|\mathbf{h}_{0}\right\|^{2}+\ldots+\left\|\mathbf{h}_{L}\right\|^{2}=\operatorname{Tr}\left(\boldsymbol{H}^{T} \boldsymbol{H}\right)$. Luego, los filtros

\footnotetext{
${ }^{10}$ Esta correspondencia tiene sentido pues generalmente se utilizan ventanas positivas, es decir, tales que $w_{n}>0$ para todo $n \in \mathcal{N}$.

${ }^{11}$ La misma puede hallarse fácilmente mediante el método de multiplicadores de Lagrange.
}

de Taylor-Fourier son soluciones al siguiente problema de optimización:

$$
\begin{aligned}
& \min _{\boldsymbol{H}} \operatorname{Tr}\left(\boldsymbol{H}^{T} \boldsymbol{H}\right) \\
& \text { sujeto a } \boldsymbol{H}^{\boldsymbol{T}} \boldsymbol{N}=\boldsymbol{I} .
\end{aligned}
$$

Análogamente a la generalización que se planteó para interpretar al estimador WDFT, el estimador WTFT se obtiene resolviendo el siguiente problema de optimización, que generaliza el problema anterior

$$
\begin{aligned}
& \min _{\boldsymbol{H}} \operatorname{Tr}\left(\boldsymbol{H}^{T} \boldsymbol{\Sigma} \boldsymbol{H}\right) \\
& \text { sujeto a } \boldsymbol{H}^{\boldsymbol{T}} \boldsymbol{N}=\boldsymbol{I},
\end{aligned}
$$

donde en este caso hacemos la asociación $\boldsymbol{\Sigma}=\left(\boldsymbol{W}^{T} \boldsymbol{W}\right)^{-1}$. De hecho, la solución a este problema es ${ }^{12}$

$$
\boldsymbol{H}=\boldsymbol{\Sigma}^{-1} \boldsymbol{N}\left(\boldsymbol{N}^{T} \boldsymbol{\Sigma}^{-1} \boldsymbol{N}\right)^{-1},
$$

lo cual produce los estimadores

$$
\widehat{\mathbf{X}}=\left(\boldsymbol{N}^{T} \boldsymbol{\Sigma}^{-1} \boldsymbol{N}\right)^{-1} \boldsymbol{N}^{T} \boldsymbol{\Sigma}^{-1} \mathbf{y},
$$

que generaliza la Ec. (48). Por último, es interesante notar que los problemas (74) y (75) se pueden desacoplar fácilmente en $L+1$ problemas independientes, lo cual permite optimizar la resolución del problema en una forma similar a la que se plantea en [30].

\section{CONCLUSIOnES}

En este trabajo hemos presentado un resumen de algunos de los estimadores de sincrofasores más importantes que se han desarrollado desde que el problema cobró interés en los años 80 y, más importante aún, la conexión entre ellos. En primer lugar, analizamos los algoritmos más simples basados en un modelo de fasor constante $\mathrm{y}$, en forma más general, en un modelo de fasor polinómico. Luego, presentamos los estimadores CSIP, un enfoque novedoso basado en optimización semi-infinita convexa que ofrece una gran flexibilidad de diseño. Concretamente, permite incluir precisamente las restricciones que debe satisfacer el sistema en el dominio del tiempo y de la frecuencia. En particular, esto permite controlar tanto el comportamiento del estimador en frecuencia no nominal, como los sobrepi$\cos$, de modo que resuelve los problemas que afectan a los estimadores basados en la DFT y la TFT. Hemos finalizado el trabajo mostrando que existe una conexión, a través de la formulación de problemas de estimación de cuadrados mínimos con distintas restricciones, entre los estimadores DFT, WDFT, TF, WTF, y CSIP. Se espera que en el desarrollo futuro de los estimadores de sincrofasores, se propongan diversos esquemas adaptivos partiendo de filtros prototipos diseñados con el enfoque CSIP, con el fin de relajar las restricciones del problema original en pos de un mejor desempeño en general. En el presente estamos trabajando en esta temática interesante.

\footnotetext{
${ }^{12}$ Nuevamente el problema se puede resolver mediante multiplicadores de Lagrange, planteando en este caso una matriz $\boldsymbol{\Lambda}$ de multiplicadores de Lagrange. La función de Lagrange en este caso sería, por ejemplo,

$$
\mathcal{L}(\boldsymbol{H}, \boldsymbol{\Lambda})=\operatorname{Tr}\left(\boldsymbol{H}^{T} \boldsymbol{\Sigma} \boldsymbol{H}\right)+\operatorname{Tr}\left(\boldsymbol{\Lambda}\left(\boldsymbol{I}-\boldsymbol{H}^{T} \boldsymbol{N}\right)\right) .
$$
}




\section{AGRADECIMIENTOS}

El trabajo de F. Messina es financiado por una beca de doctorado Peruilh de la Facultad de Ingeniería de la Universidad de Buenos Aires. Este artículo fue posible por la financiación del proyecto FONARSEC UREE 4 "Sistema de Medición fasorial orientado al desarrollo de redes inteligentes" del FONARSEC, Ministerio de Ciencia, Tecnología e Innovación Productiva; y el proyecto PIP11220150100578CO del CONICET.

\section{REFERENCIAS}

[1] G. Giannakis, V. Kekatos, N. Gatsis, S.-J. Kim, H. Zhu, and B. Wollenberg, "Monitoring and Optimization for Power Grids: A Signal Processing Perspective," IEEE Signal Process. Mag, vol. 30, no. 5, pp. 107-128, Sept. 2013.

[2] F. Aminifar, M. Fotuhi-Firuzabad, A. Safdarian, A. Davoudi, and M. Shahidehpour, "Synchrophasor measurement technology in power systems: Panorama and state-of-the-art," IEEE Access, vol. 2, pp. 1607-1628, 2014.

[3] J. D. L. Ree, V. Centeno, J. S. Thorp, and A. G. Phadke, "Synchronized phasor measurement applications in power systems," IEEE Transactions on Smart Grid, vol. 1, no. 1, pp. 20-27, June 2010.

[4] IEEE Standard for Synchrophasor Measurements for Power Systems, IEEE Std. C37.118.1-2011 (Revision of IEEE Std C37.118-2005), Dec. 2011.

[5] IEEE Standard for Synchrophasor Measurements for Power Systems Amendment 1: Modification of Selected Performance Requirements, IEEE Std. C37.118.1a-2014 (Amendment to IEEE Std C37.118.12011), Apr. 2014.

[6] P. Castello, M. Lixia, C. Muscas, and P. Pegoraro, "Impact of the Model on the Accuracy of Synchrophasor Measurement," IEEE Trans. Instrum. Meas., vol. 61, no. 8, pp. 2179-2188, Aug. 2012.

[7] G. Barchi, D. Macii, and D. Petri, "Synchrophasor estimators accuracy: A comparative analysis," IEEE Trans. Instrum. Meas., vol. 62, no. 5, pp. 963-973, May 2013.

[8] G. Barchi, D. Macii, D. Belega, and D. Petri, "Performance of synchrophasor estimators in transient conditions: A comparative analysis," IEEE Trans. Instrum. Meas., vol. 62, no. 9, pp. 2410-2418, Sept 2013.

[9] M. Karimi-Ghartemani and H. Karimi, "Processing of symmetrical components in time-domain," IEEE Trans. Power Syst., vol. 22, no. 2 , pp. 572-579, May 2007

[10] J. Grainger and W. Stevenson, Power System Analysis. McGraw-Hill, 1994.

[11] F. Messina, P. Marchi, L. Rey Vega, C. G. Galarza, and H. Laiz, "A Novel Modular Positive-Sequence Synchrophasor Estimation Algorithm for PMUs," IEEE Transactions on Instrumentation and Measurement, vol. 66, no. 6, pp. 1164-1175, June 2017.

[12] A. Phadke and J. Thorp, Synchronized Phasor Measurements and Their Applications, ser. Power Electronics and Power Systems. Springer US, 2008.

[13] S. M. Kay, Fundamentals of Statistical Signal Processing: Estimation Theory. Upper Saddle River, NJ, USA: Prentice-Hall, Inc., 1993.

[14] G. Turin, "An introduction to matched filters," IRE Transactions on Information Theory, vol. 6, no. 3, pp. 311-329, June 1960.

[15] A. Oppenheim and R. Schafer, Discrete-Time Signal Processing, ser. Prentice-Hall signal processing series. Pearson Education, 2011.

[16] D. Macii, D. Petri, and A. Zorat, "Accuracy of dft-based synchrophasor estimators at off-nominal frequencies," in 2011 IEEE International Workshop on Applied Measurements for Power Systems (AMPS), Sept 2011, pp. 19-24.

[17] G. Andria, M. Savino, and A. Trotta, "Windows and interpolation algorithms to improve electrical measurement accuracy," IEEE Transactions on Instrumentation and Measurement, vol. 38, no. 4, pp. 856863, Aug 1989.

[18] C. Offelli and D. Petri, "The influence of windowing on the accuracy of multifrequency signal parameter estimation," IEEE Transactions on Instrumentation and Measurement, vol. 41, no. 2, pp. 256-261, Apr 1992.

[19] P. Stoica and R. Moses, Spectral Analysis of Signals. Pearson Prentice Hall, 2005. [Online]. Available: https://books.google.com.ar/books?id=h78ZAQAAIAAJ

[20] D. Macii, D. Petri, and A. Zorat, "Accuracy Analysis and Enhancement of DFT-Based Synchrophasor Estimators in Off-Nominal Conditions," IEEE Trans. Instrum. Meas., vol. 61, no. 10, pp. 2653 2664, Oct. 2012.
[21] D. Belega and D. Petri, "Accuracy Analysis of the Multicycle Synchrophasor Estimator Provided by the Interpolated DFT Algorithm," IEEE Trans. Instrum. Meas., vol. 62, no. 5, pp. 942-953, May 2013.

[22] D. Belega, D. Dallet, and D. Petri, "Statistical description of the sinewave frequency estimator provided by the interpolated dft method," Measurement, vol. 45, no. 1, pp. 109 - 117, 2012. [Online]. Available: http://www.sciencedirect.com/science/article/pii/S0263224111003058

[23] J. A. de la O Serna, "Dynamic Phasor Estimates for Power System Oscillations," IEEE Trans. Instrum. Meas., vol. 56, no. 5, pp. 16481657, Oct. 2007.

[24] M. A. Platas-Garza and J. A. de la O Serna, "Dynamic Phasor and Frequency Estimates Through Maximally Flat Differentiators," IEEE Transactions on Instrumentation and Measurement, vol. 59, no. 7, pp. 1803-1811, July 2010.

[25] D. Petri, D. Fontanelli, and D. Macii, "A frequency-domain algorithm for dynamic synchrophasor and frequency estimation," IEEE Trans. Instrum. Meas., vol. 63, no. 10, pp. 2330-2340, Oct. 2014.

[26] F. Messina, L. Rey Vega, P. Marchi, and C. G. Galarza, "Optimal Differentiator Filter Banks for PMUs and their Feasibility Limits," IEEE Transactions on Instrumentation and Measurement, 2017, en prensa.

[27] D. Belega, D. Fontanelli, and D. Petri, "Dynamic phasor and frequency measurements by an improved taylor weighted least squares algorithm," IEEE Transactions on Instrumentation and Measurement, vol. 64, no. 8, pp. 2165-2178, Aug 2015.

[28] J. A. de la O Serna, "Synchrophasor measurement with polynomial phase-locked-loop taylor-fourier filters," IEEE Transactions on Instrumentation and Measurement, vol. 64, no. 2, pp. 328-337, Feb 2015.

[29] M. A. Platas-Garza and J. A. de la O Serna, "Dynamic harmonic analysis through taylor-fourier transform," IEEE Transactions on Instrumentation and Measurement, vol. 60, no. 3, pp. 804-813, March 2011.

[30] $\stackrel{2011 .}{-}$,Polynomial implementation of the taylor-fourier transform for harmonic analysis," IEEE Transactions on Instrumentation and Measurement, vol. 63, no. 12, pp. 2846-2854, Dec 2014.

[31] A. Jerri, The Gibbs Phenomenon in Fourier Analysis, Splines and Wavelet Approximations, ser. Mathematics and Its Applications. Springer US, 1998. [Online]. Available: https://books.google.com.ar/books?id=y2c00LAMnFUC

[32] D. Macii, G. Barchi, and D. Petri, "Design criteria of digital filters for synchrophasor estimation," in 2013 IEEE International Instrumentation and Measurement Technology Conference (I2MTC), May 2013, pp. 1579-1584.

[33] A. J. Roscoe, B. Dickerson, and K. E. Martin, "Filter design masks for c37.118.1a-compliant frequency-tracking and fixed-filter m-class phasor measurement units," IEEE Trans. Instrum. Meas., vol. 64, no. 8, pp. 2096-2107, Aug 2015.

[34] F. Messina, P. Marchi, L. Rey Vega, and C. G. Galarza, "Design of Synchrophasor Estimation Systems with Convex Semi-Infinite Programming," in Electrical Power and Energy Conference (EPEC), Ottawa, Canada, Oct. 2016.

[35] A. W. Potchinkov, "Design of optimal linear phase fir filters by a semiinfinite programming technique," Signal Processing, vol. 58, no. 2, pp. 165-180, 1997

[36] R. Reemtsen and J. Rückmann, Semi-Infinite Programming, ser. Nonconvex Optimization and Its Applications. Springer US, 1998.

[37] S. Boyd and L. Vandenberghe, Convex Optimization, ser. Berichte über verteilte messysteme. Cambridge University Press, 2004.

[38] MATLAB, Optimization Toolbox. Natick, Massachusetts: The MathWorks Inc., 2016.

[39] "Semi-infinite programming solvers: CSIP and NSIPS," http://www.norg.uminho.pt/aivaz/software.html. 